\title{
chemosensors
}

ISSN 2227-9040

www.mdpi.com/journal/chemosensors

Article

\section{Chemo-Electrical Signal Transduction by Using Stimuli-Responsive Polymer Gate-Modified Field Effect Transistor}

\author{
Akira Matsumoto ${ }^{1, \dagger}$, Yusuke Tsurui ${ }^{2, \dagger}$, Hiroko Matsumoto ${ }^{1}$, Yasuhiro Maeda ${ }^{1}$, Toru Hoshi ${ }^{2}$, \\ Takashi Sawaguchi $^{2}$ and Yuji Miyahara ${ }^{1, *}$
}

1 Institute of Biomaterials and Bioengineering, Tokyo Medical and Dental University, 2-3-10 Kanda-Surugadai, Chiyoda-ku, Tokyo 101-0062, Japan;

E-Mails: matsumoto.bsr@tmd.ac.jp (A.M.); hiroko.matsumoto.bsr@tmd.ac.jp (H.M.); miyahara.bsr@tmd.ac.jp (Y.M.)

2 Department of Materials and Applied Chemistry, College of Science and Technology, Nihon University, 1-8-14 Kanda-Surugadai, Chiyoda-ku, Tokyo 101-8308, Japan; E-Mails: csyu09143@g.nihon-u.ac.jp (Y.T.); hoshi.toru@nihon-u.ac.jp (T.H.); sawaguchi.takashi@nihon-u.ac.jp (T.S.)

$\dagger$ These authors contributed equally to this work.

* Author to whom correspondence should be addressed; E-Mail: miyahara.bsr@tmd.ac.jp; Tel./Fax: +81-3-5280-8095.

Received: 8 October 2013; in revised form: 10 January 2014 / Accepted: 13 March 2014 / Published: 26 March 2014

\begin{abstract}
A glucose-responsive polymer brush was designed on a gold electrode and exploited as an extended gate for a field effect transistor (FET) based biosensor. A permittivity change at the gate interface due to the change in hydration upon specific binding with glucose was detectable. The rate of response was markedly enhanced compared to the previously studied cross-linked or gel-coupled electrode, owing to its kinetics involving no process of the polymer network diffusion. This finding may offer a new strategy of the FET-based biosensors effective not only for large molecules but also for electrically neutral molecules such as glucose with improved kinetics.
\end{abstract}

Keywords: field effect transistors; permittivity; Debye length; stimuli-responsive polymers 


\section{Introduction}

Rapid, sensitive and economically acceptable means to determine biological substances is of interest in all scenes of analytical science and clinical diagnosis. Field effect transistor (FET) based molecular detection provides a solid option to achieve this goal. FET is a type of transistors that modulates the source-drain "channel" conductivity in response to the change in electric field along a perpendicular direction to the gate electrode. For bio-sensing applications, bio-recognition events of interest are predesigned on the gate surface. During these events, any observed changes in the channel conductivity can be correlated to polarity and density of charges at the gate, thus providing a basis for identification and quantification of these molecules. This strategy, often termed "bio-FETs", has been validated for a variety of targets [1-4] including ions [1], enzymes [5], proteins [6,7], nucleotides [8-11] carbohydrates [12,13] and even cells [14-21]. The beauty of bio-FETs may be highlighted in the fact that it represents a label-free determination platform, which can be readily downsized and integrated by virtue of today's advanced semiconductor fabrication technology. While offering a number of applications, the strategy of bio-FETs often finds difficulty in dealing with relatively large molecules such as proteins. This intrinsic limitation is related to the thickness of electrical double layer or the Debye length formed at the gate surface, which is no greater than a few nanometers in length under physiological ionic conditions. Beyond this length, counterion-screening effect prevails masking otherwise visible molecular charges and, as a result, the technique yields an upper limit of the molecular size for which the charge sensing can be reliably performed [22,23].

To cope with this limitation, we have previously described a role of stimuli-responsive polymer gels (or smart gels) to enable the "Debye-length-free" signal transduction [24-26]. When configured as a thin coat on the gate electrode, smart gels could serve as a modulator of the interfacial permittivity upon detection of each specific target due to the change in hydration, a parameter also detectable by the principle of bio-FET [24,25]. Owing to the ability of smart gels to spatially propagate the signal (synchronized with the change in hydration) across a macroscopic scale of distance, the technique was virtually free from the Debye length. However, for a critical shortcoming, this mode of the signal propagation was significantly slow, the rate of which was determined by cooperative diffusion of the polymer network, typically requiring tens of minutes to complete.

This work focuses on the use of a linear or non-crosslinked polymer system in order to exclude such a slow (rate-determining) process. For this purpose, the gate surface was modified with a newly synthesized phenylboronate-functionalized glucose-responsive polymer. This polymer was capable of undergoing reversible, abrupt and glucose-dependent change in hydration at room temperature, providing a convenient platform to demonstrate such a way of signal-transduction with remarkably improved rate of response.

\section{Experimental Section}

\subsection{Materials}

N-isopropylacrylamide (NIPAAm), azobisisobutyronitrile (AIBN) and cupper bromide(I) were purchased from Wako Pure Chemical Industries, Ltd. (Osaka, Japan). Pentandiethylenetriamine was purchased from Tokyo Chemical Industry (Tokyo, Japan). Tris(2-aminoethyl)amine, ethyl 
$\alpha$-bromoisobutyrate and bis[2-(2-bromoisobutyryloxy)-undecyl]disulfide were purchased form Aldrich Chem. Co. (Milwaukee, WI, USA). N-isopropylacrylamide was recrystallized in hexane before use. Azobisisobutyronitrile was recrystallized in methanol. All other chemicals were used as received. 4-(2-acrylamidoethylcarbamoyl)-3-fluorophenylboronic acid (AECFPBA) was synthesized as reported previously [27].

\subsection{Preparation of Gold Extended Gate Electrode for FET and the Surface Modification}

A synthetic quartz substrate $(\mathrm{Ra}=1.8 \mathrm{~nm})$ bearing 10 islands of sputtered polycrystalline-gold layer $(100 \mathrm{~nm})$ using titanium as an adhesion layer $(10 \mathrm{~nm})$ was used as an extended gate for Bio-FET. The specific thickness of each layer was obtained by the controlled sputter-exposure times.

For convenience in the following surface modification, a glass tube with the inner diameter of $5 \mathrm{~mm}$ was installed using a thermosetting insulating resin (ZC-203, Nippon Pelnox, Tokyo, Japan) to surround the area of the electrodes. The inner area of the chamber was plasma-cleaned in an oxygen plasma reactor (PDC 210, Yamato, Japan) for $60 \mathrm{~s}$ with the power of $300 \mathrm{~W}$ under the oxygen pressure of $150 \mathrm{~Pa}$, prior to use. The cleaned electrode surface was immersed in a $10 \mathrm{mM}$ ethanol solution containing bis[2-(2-bromoisobutyryloxy)-undecyl]disulfide to install the surface with bromine group terminated self-assembled monolayer (SAM) for the following surface initiated ATRP. The same molar amount of dithiothreitol (DTT) was also added for the reduction of the disulfide group into the thiol group, to facilitate the rate of thiol-Au chemo-sorption reaction. After $24 \mathrm{~h}$ incubation at room temperature the modified electrode surface was thoroughly washed by sonication in pure ethanol. These surface modification procedures are schematically depicted in Figure 1a.

Figure 1. (a) Fabrication route of polymer modified gold electrode. (b) Circuit diagram for field effect transistor (FET) based measurement.
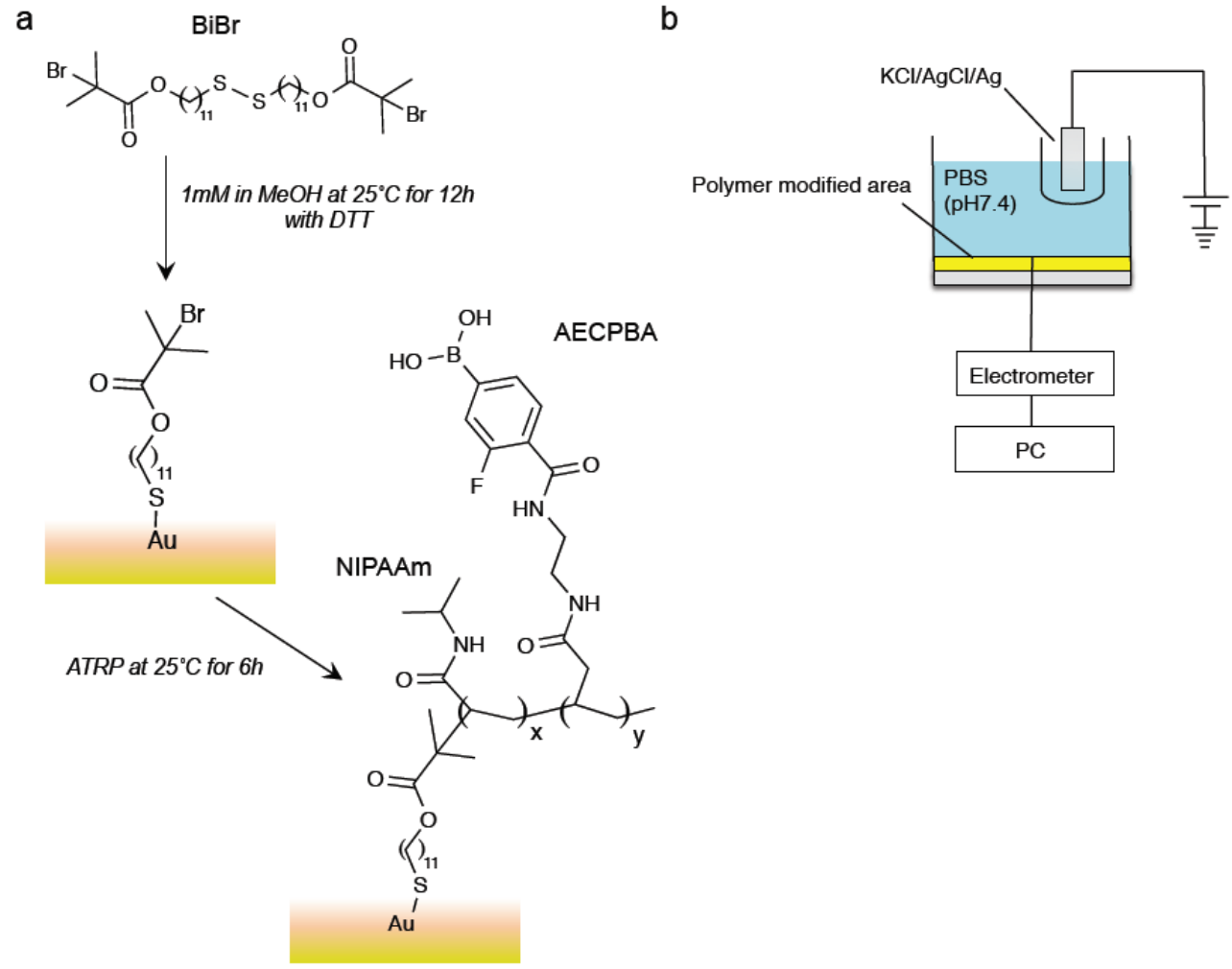
The packing density of the SAM was assessed by cyclic voltammetry (CV) using an Autolab PGSTAT 302 (Eco Chemie, Utrecht, The Netherlands) potentiostat equipped with a three-electrode system using a platinum wire as a counter electrode. The voltage was scanned from -0.2 to $-1.35 \mathrm{~V}$ (vs. reference $\mathrm{Ag} / \mathrm{AgCl}$ electrode) at the rate of $0.5 \mathrm{~V} / \mathrm{s}$ with resolution of $0.5 \mathrm{mV}$ in an aqueous $0.5 \mathrm{M}$ $\mathrm{KOH}$ solution. The first scan reduction peak found at the range of -0.8 to $-1.0 \mathrm{~V}$ was attributed to the charge transfer from the electrode to the thiol. The number of thiol groups per unit area $\left(\gamma, \mathrm{nm}^{-2}\right)$ was calculated as:

$$
\gamma=Q_{S A M} N_{A} / F
$$

where $Q_{S A M}$ is the total surface charge density calculated from the background corrected peak area, scan rate and the electrode surface area, $N_{A}$ the Avogadro's constant and $F$ the Faraday constant [28]. The density of the SAM was determined to be $3.1 \pm 0.7$ chains $\mathrm{nm}^{-2}$, which is comparable to those reported and what is theoretically expected when the maximum coverage on the Au (111) surface occurs. Thus, we concluded that the cleavage of disulfide bonds of bis[2-(2-bromoisobutyryloxy)undecyl]disulfide and the subsequent $\mathrm{Au}-\mathrm{S}$ chemisorption on the electrode occurred in an almost stoichiometric manner.

\subsection{Synthesis of Poly(NIPAAm-Co-AECPBA)}

Under an argon atmosphere, a mixed solvent DMF/water (80:20, v/v, $1 \mathrm{~mL})$, NIPAAm, AECFPBA, $\mathrm{CuBr}(92.5 \mu \mathrm{mol})$, and PMDETA $(278 \mu \mathrm{mol})$ were charged into the flask, which was tightly sealed with a rubber septum. The reaction mixture was degassed by three freeze-vacuum-thaw cycles, which was poured onto the above-described electrode chamber under an argon atmosphere. To an identical reaction mixture, ethyl $\alpha$-bromoisobutyrate $(92.5 \mu \mathrm{mol})$ was added as a soluble initiator to allow the solution-phase ATRP, separately form the surface-initiated reaction. This experiment was meant to provide an indirect characterization of the gate-surface-introduced polymers. Each reaction was allowed with constant stirring at room temperature for $1 \mathrm{~h}$, which was then terminated by exposing to the air. The obtained solution (from the solution-phase ATRP) was passed through a basic alumina column. The eluted fraction was further dialyzed against purified water for 2 days and finally freeze-dried. The yield, fraction of AECFPBA as determined by ${ }^{1} \mathrm{H}-\mathrm{NMR}, M_{\mathrm{n}}$ and $M_{\mathrm{w}} / M_{\mathrm{n}}$ as determined by GPC for all polymers are summarized in Table 1 . The copolymer compositions were determined by ${ }^{1} \mathrm{H}-\mathrm{NMR}$ $\left(500 \mathrm{MHz}, \mathrm{D}_{2} \mathrm{O}\right)$ from the peak intensity ratios of methine proton on isopropyl group of NIPAAm $(\delta 3.8 \mathrm{ppm})$ relative to those of aromatic protons of AECPBA $(\delta$ 7.3-7.6) (SI Figure 1). All polymers prepared in solution phase had $M_{\mathrm{n}}$ of about 2000 with reasonably narrow distribution $\left(M_{\mathrm{w}} / M_{\mathrm{n}}=1.3\right)$ regardless of the fraction of AECFPBA, which is indicative of quantitative progress of ATRP. The morphology of the polymer-modified electrode was investigated using a Scanning Electron Microscope (S-3400N, Hitachi Science Systems, Ltd., Tokyo, Japan) with the accelerating voltage of $2 \mathrm{kV}$. Samples were first mounted on carbon fiber pads preadhered to aluminum stubs. Images were taken and analyzed by the equipped software (SI Figure 2). 
Table 1. Preparation and characterization of PBA containing polymer.

\begin{tabular}{cccccc}
\hline Abbreviation & NIPAAm [wt \% $^{\mathbf{1}}$ & AECFPBA [wt \% $^{\mathbf{2}}$ & Mn & Mw/Mn & Yield [wt\%] \\
\hline PNIP-B-10 & 90 & 10 & $1.3 \times 10^{3}$ & 1.3 & 30.0 \\
PNIP-B-20 & 80 & 20 & $1.2 \times 10^{3}$ & 1.4 & 30.0 \\
PNIP-B-30 & 70 & 30 & $1.2 \times 10^{3}$ & 1.3 & 17.9 \\
\hline
\end{tabular}

${ }^{1}$ Molar fraction of NIPAAm in feed. ${ }^{2}$ Molar fraction of AECFPBA in feed.

\subsection{Transmittance Measurement for Determination of Lower Critical Solution Temperature}

The LCSTs (Lower Critical Solution Temperatures) of the $0.1 \mathrm{wt} \%$ polymer solutions for various glucose concentrations were assessed by observing the change in transmittance at $500 \mathrm{~nm}$ using a UV-VIS spectrophotometer (UV-2450, Shimadzu Co. Ltd., Kyoto, Japan). Each sample was set in a thermostatic cell holder and heated at the rate of $0.2{ }^{\circ} \mathrm{C} / \mathrm{min}$. The LCST was defined as the temperature at the inflection point in the transmittance versus temperature curves (SI Figure 3).

\subsection{Measurement of Surface Potential}

The prepared 10-channel gold electrode was electrically lined to a Keithley 6517B electrometer/high resistance meter (Keithley, Cleveland, OH, USA) through a switching circuit (Figure $1 b)$. Drain current (Id), drain-source voltage (Vds), and biased gate voltage $\left(\mathrm{V}_{\mathrm{G}}\right)$ were $600 \mu \mathrm{A}$, $1.0 \mathrm{~V}$, and $0 \mathrm{~V}$ (versus $\mathrm{Ag} / \mathrm{AgCl}$ ), respectively. The reaction chamber was filled with a $50 \mathrm{mM} \mathrm{PBS}$ adjusted to $\mathrm{pH} 7.4(155 \mathrm{mM} \mathrm{NaCl})$. Changes in the surface potential were monitored when adding graded amounts of glucose in solution. All measurements were carried out at room temperature with an $\mathrm{Ag} / \mathrm{AgCl}$ reference electrode in saturated $(3.3 \mathrm{M}) \mathrm{KCl}$ solution immobilized in a salt bridge.

\section{Results and Discussion}

The focus of this study is to demonstrate the feasibility of exploiting stimuli-responsive-polymerbrushed surfaces as a route to obtain prompt signal transduction in Bio-FETs. Threshold gate voltage $\left(V_{T}\right)$ of Bio-FETs is sensitive to both charge density $(Q)$ and capacitance $(C)$ of the gate, and their relationship is qualitatively expressed as:

$$
\Delta V_{T}=\Delta Q / \Delta C
$$

Due to a marked discrepancy in the relative permittivity between water ( $c a$. 80) and organic materials ( $c a .2$ ), when the gate-introduced material undergoes abrupt change in hydration, it leads to modulation of $\Delta C$ and thus $\Delta V_{T}$. Our previous reports had shown that the direction of $\Delta V_{T}$ of the smart gel-coupled bio-FETs was actively controllable depending on the material's polarity and magnitude of the change in hydration [25]. While these observations offered a rationale for the "Debye-length-free" signal-transduction, its kinetics was significantly slow due to the rate-determining process of cooperative network diffusion inherent to the cross-linked (gel) system.

As a platform polymer to decorate the gate, PNIPAAm main-chained copolymers bearing PBA functionality were newly synthesized. The pendent PBA is able to reversibly bind with glucose and dynamically change the state of equilibrium, as shown in Figure 2a. With increase of glucose concentration, the fraction of hydrophilic phenylboronate increases, thus increasing the overall 
solubility of the polymer [29]. This feature has been summarized in Figure 2b, where a glucose-induced increment of lower critical solution temperatures (LCST) has been systemically revealed for different contents of PBA. It should be noted that PBA is unique in that it undergoes a dramatic inversion in its level of hydrophobicity depending on the degree of acid disassociation; it is strongly hydrophobic when uncharged (Figure 2a, (i)) but it becomes hydrophilic when negatively charged (Figure 2a, (ii) and (iii)). Therefore, an increase of the PBA content in the copolymer, on the one hand, results in a decreased LCST in the absence of glucose, on the other hand, leads to more profound glucose-dependent change, as observed in Figure 2b. Our first effort was to gain insight into an optimal fraction of PBA, with which the polymer would show dramatic glucose-dependent solubility changes at around room temperature $\left(25^{\circ} \mathrm{C}\right)$. Based on the results obtained in Figure 2b, PNIP-B-20 and PNIP-B-30 (20 and $30 \mathrm{w} / v \%$ of PBA in feed, respectively) were chosen as structures to be introduced to the gate surface. These polymers show LCSTs of $21{ }^{\circ} \mathrm{C}$ and $25{ }^{\circ} \mathrm{C}$, respectively, at $1 \mathrm{~g} / \mathrm{L}$ of glucose, which is an equivalent concentration to human normoglycemia (Figure 2b). Also disclosed in Figure $2 \mathrm{~b}$ is that the LCST of these polymers dramatically increases with further increase of the glucose concentration, and vice versa. The equivalent polymers (to PNIP-B-20 and PNIP-B-30) were introduced to gold electrodes by conducting the surface-initiated ATRP as described above. For controls, non-modified (bare Au) electrodes and those modified with PNIPAAm homopolymer were prepared.

Figure 2. (a) Glucose-dependent equilibria of PBA. (b) Glucose dependent changes in the lower critical solution temperatures (LCST) of PNIP-B for different contents of PBA.

a

(i)

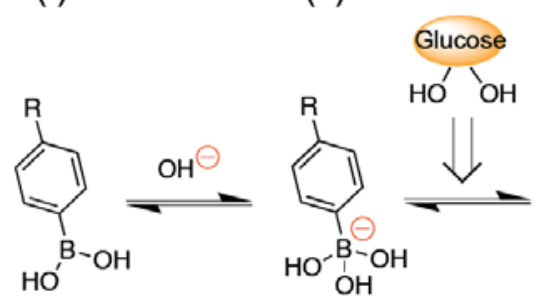

b

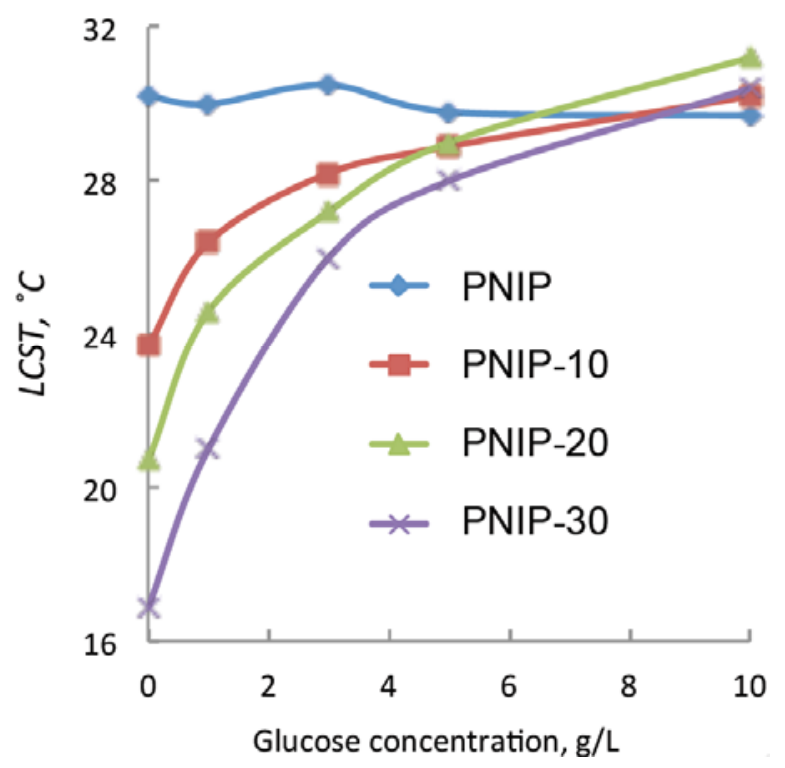

The polymer modified gold electrodes were electrically lined to an electrometer and were assessed for their potentiometric response to glucose. Figure 3 shows changes in the surface potential of the PNIP-B-30 modified electrode as a function of the glucose concentration investigated at $25{ }^{\circ} \mathrm{C}$. When increasing the glucose concentration from hypo- to normo- and hyperglycemic levels, the potential increased as large as $20 \mathrm{mV}$. Importantly, this positive direction shift of the potential indicates dominant contribution of the polymer hydration, rather than an increase of the phenylboronate anions when binding with glucose. As for the selectivity, PBA can also bind with other 1.2 or 1.3 cis-diols 
including other sugars, catechol, RNA and etc. Indeed, the binding constant between PBA and glucose is comparable to those for other naturally occurring monosaccharides such as mannose and galactose [30]. Even so, in consideration of their respective blood concentrations, in practice, it can be regarded as glucose specific, as demonstrated by many other researches. Because of a lack in the number of experiments, the detection limit of glucose in the present configuration is yet to be determined. As a reference, our previous study had shown that a gold electrode modified with a self-assembled monolayer of PBA could accomplish a potentiometric (charge) detection of glucose with its detection limit of $0.2 \mathrm{~g} / \mathrm{L}$, which is safely below normoglycemia, i.e., $1 \mathrm{~g} / \mathrm{L}$ [31].

Figure 3. Surface potential changes of PNIP-B-30 modified electrode as a function of the glucose concentration investigated at $25^{\circ} \mathrm{C}$. Data are expressed as averages + S.D. $(n=10)$.

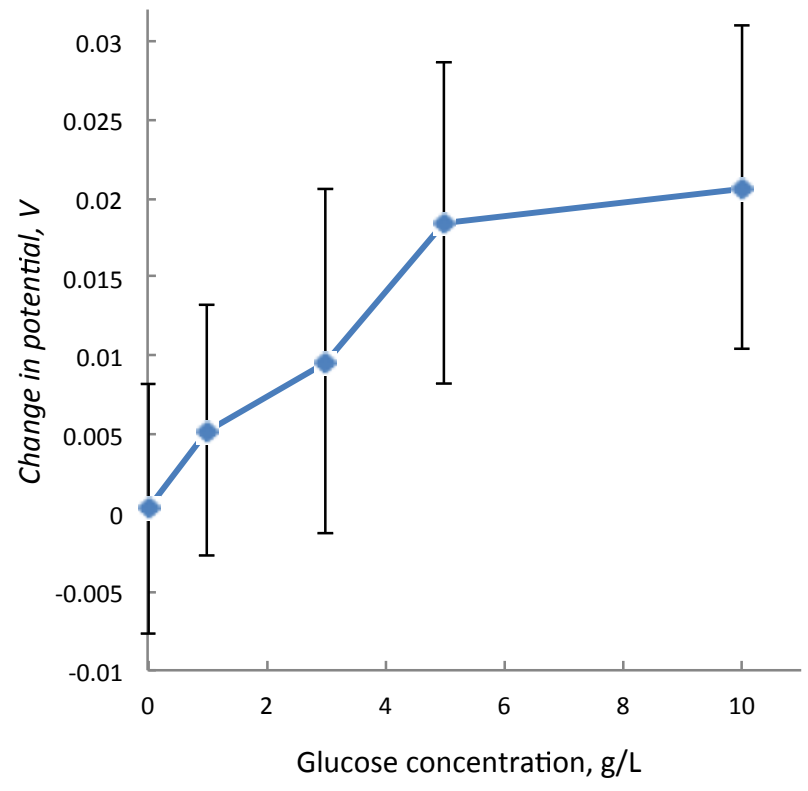

Representatively shown in Figure 4 is a kinetic feature of the potentiometric response of the PNIP-B-20-modified electrode when adding $10 \mathrm{~g} / \mathrm{L}$ glucose at $27.5^{\circ} \mathrm{C}$, along with that of the control (PNIPAAm-modified electrode). A remarkably elevated potential (as large as $100 \mathrm{mV}$ ) is evident specifically for the polymer-modified electrode; the magnitude of the potential change is much greater than that obtained in Figure 3 where the layer was composed of PNIP-B-30 and recorded at $25{ }^{\circ} \mathrm{C}$. This difference may be ascribed to that in the initial state of the hydration (with no glucose) specific to the type of polymer and each investigated temperature, which is characteristic of the amphiphilic polymer-coupled Bio-FETs. Furthermore, Figure 4 also demonstrates a remarkably enhanced rate of response, in contrast to the previously reported cross-linked polymer systems; the early stage response in Figure 4 appears to complete within a few minutes, while the gel-coupled systems typically required several tens of minutes. This enhancement in the rate of response may be accounted for by distinct kinetics of the polymer chain hydration free from the cooperative polymer chain diffusion, a rate-determining process for the gel-combined systems. 
Figure 4. Time-course change in the surface potential of gold electrodes modified with PNIP-B-20 (red line) and PNIP (blue line) on addition of $10 \mathrm{~g} / \mathrm{L}$ glucose at $27.5{ }^{\circ} \mathrm{C}$.

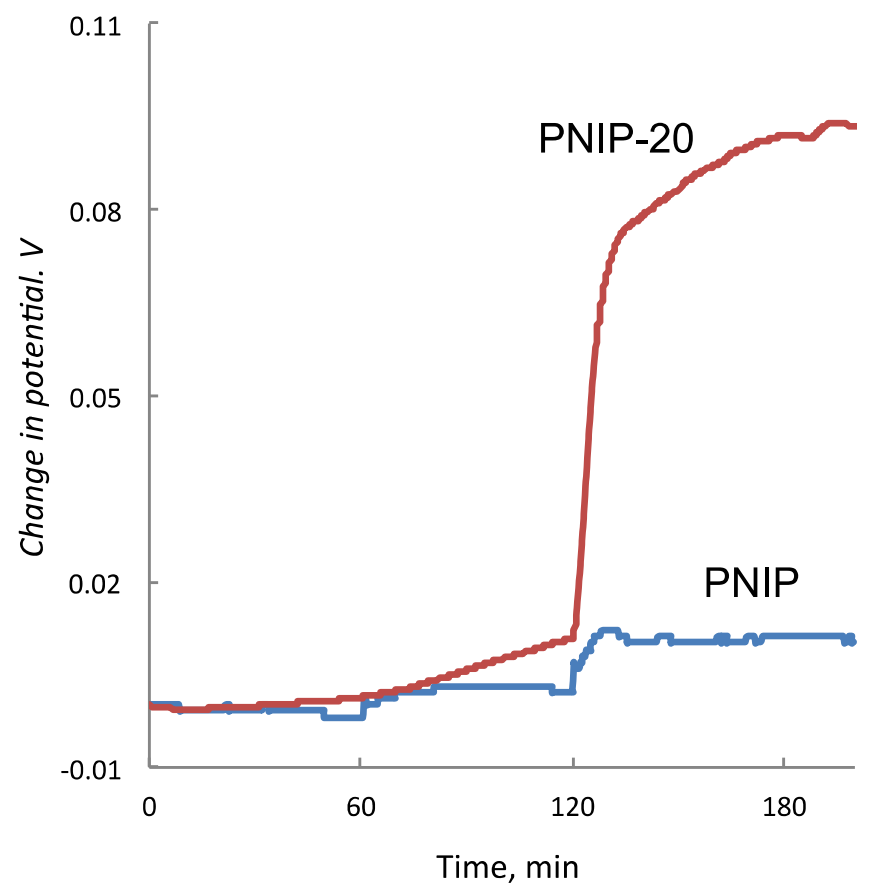

\section{Conclusions}

Our results demonstrates that a stimuli-responsive polymer brushed surface exhibiting target-specific change in hydration can provide a rationale for the "Debye length-free" signal-transduction for Bio-FETs. In comparison to previously studied cross-linked (or gel-based) interfaces, a markedly enhanced rate of response was achievable, owing to its kinetics that is free from the process of the network diffusion. Noteworthy is that many other stimuli-responsive polymers are available that are responsive to other (than glucose) biologically important targets including proteins and biomarkers [32,33]. The strategy described in this paper may also be effective to those targets. Thus, this finding offers additional prospects for its use as a universal platform of Bio-FETs applicable to large molecules and even those electrically neutral such as glucose.

\section{Acknowledgments}

This study was supported by Japan Science and Technology Agency (JST), Core Research of Evolutional Science \& Technology (CREST) and Japan Society for the Promotion of Science (JSPS) through a grant-in-aid for young scientists (A), and, in part, by JSPS through the "Funding Program for World-Leading Innovative R\&D on Science and Technology (FIRST Program)," initiated by the Council for Science and Technology Policy (CSTP). The fabrication of gold electrode was supported by the Nano-Integration Facility at the National Institute for Materials Science (NIMS).

\section{Conflict of Interest}

The authors declare no conflict of interest. 


\section{References}

1. Bergveld, P. Development of an ion-sensitive solid-state device for neurophysiological measurements. IEEE Trans. Biomed. Eng. 1970, 17, 70-71.

2. Schoning, M.J.; Poghossian, A. Recent advances in biologically sensitive field-effect transistors (BioFETs). Analyst 2002, 127, 1137-1151. References are therein.

3. Matsumoto, A.; Miyahara, Y. Current and emerging challenges in field effect transistor based bio-sensing. Nanoscale 2013, 5, 10702-10718. References are therein.

4. Janata, J.; Moss, S.D. Chemically sensitive field-effect transistors. Biomed. Eng. 1976, 11, 241-245.

5. Caras, S.; Janata, J. Field-Effect transistor sensitive to penicillin. Anal. Chem. 1980, 52, 1935-1937.

6. Schasfoort, R.B.M.; Kooyman, R.P.H.; Bergveld, P.; Greve, J. A New Approach to Immunofet Operation. Biosens. Bioelectron. 1990, 5, 103-124.

7. Feng, C.L.; Xu, Y.H.; Song, L.M. Study on highly sensitive potentiometric IgG immunosensor. Sens. Actuators B Chem. 2000, 66, 190-192.

8. Miyahara, Y.; Sakata, T.; Matsumoto, A. Microbial genetic analysis based on field effect transistors. In Principles of Bacterial Detection: Biosensors, Recognition Receptors and Microsystems; Zourob, M., Elwary, S., Turner, A.P.F., Eds.; (C) Springer Science + Business Media, LLC: Philadelphia, PA, USA, 2008; Volume 14, pp. 311-337. References are therein.

9. Sakata, T.; Miyahara, Y. Potentiometric detection of single nucleotide polymorphism by using a genetic field-effect transistor. Chembiochem 2005, 6, 703-710.

10. Sakata, T.; Miyahara, Y. DNA sequencing based on intrinsic molecular charges. Angew. Chem. Int. Ed. 2006, 45, 2225-2228.

11. Rothberg, J.M.; Hinz, W.; Rearick, T.M.; Schultz, J.; Mileski, W.; Davey, M.; Leamon, J.H.; Johnson, K.; Milgrew, M.J.; Edwards, M.; et al. An integrated semiconductor device enabling non-optical genome sequencing. Nature 2011, 475, 348-352.

12. Matsumoto, A.; Sato, N.; Kataoka, K.; Miyahara, Y. Noninvasive sialic acid detection at cell membrane by using phenylboronic acid modified self-assembled monolayer gold electrode. J. Am. Chem. Soc. 2009, 131, 12022-12023.

13. Matsumoto, A.; Cabral, H.; Sato, N.; Kataoka, K.; Miyahara, Y. Assessment of tumor metastasis via direct determination of cell membrane sialic acid expression. Angew. Chem. Int. Ed. 2010, 49, 5494-5497.

14. Wolf, B.; Brischwein, M.; Baumann, W.; Ehret, R.; Kraus, M. Monitoring of cellular signalling and metabolism with modular sensor-technique: The PhysioControl-Microsystem (PCM (R)). Biosens. Bioelectron. 1998, 13, 501-509.

15. Fromherz, P.; Offenhausser, A.; Vetter, T.; Weis, J. A neuron-silicon junction-A retzius cell of the leech on an insulated-gate field-effect transistor. Science 1991, 252, 1290-1293.

16. Baumann, W.H.; Lehmann, M.; Schwinde, A.; Ehret, R.; Brischwein, M.; Wolf, B. Microelectronic sensor system for microphysiological application on living cells. Sens. Actuators B Chem. 1999, 55, 77-89. 
17. Offenhausser, A.; Knoll, W. Cell-transistor hybrid systems and their potential applications. Trends Biotechnol. 2001, 19, 62-66.

18. Ingebrandt, S.; Yeung, C.K.; Krause, M.; Offenhausser, A. Cardiomyocyte-transistor-hybrids for sensor application. Biosens. Bioelectron. 2001, 16, 565-570.

19. Yeung, C.K.; Ingebrandt, S.; Krause, M.; Offenhausser, A.; Knoll, W. Validation of the use of field effect transistors for extracellular signal recording in pharmacological bioassays. J. Pharmacol. Toxicol. Methods 2001, 45, 207-214.

20. Sakata, T.; Miyahara, Y. Noninvasive monitoring of transporter-substrate interaction at cell membrane. Anal. Chem. 2008, 80, 1493-1496.

21. Schaffhauser, D.F.; Patti, M.; Goda, T.; Miyahara, Y.; Forste, R.I.C.; Dittrich, P.S. An integrated field-effect microdevice for monitoring membrane transport in xenopus laevis oocytes via lateral proton diffusion. PLoS One 2012, 7, doi:10.1371/journal.pone.0039238.

22. Schafoort, R.B.M.; Bergveld, P.; Kooyman, R.P.H.; Greve, J. Possibilities and limitations of direct detection of protein charges by means of an immunological fielf-effect transistor. Anal. Chem. Acta 1990, 238, 323-329.

23. Stern, E.; Wagner, R.; Sigworth, F.J.; Breaker, R.; Fahmy, T.M.; Reed, M.A. Importance of the Debye screening length on nanowire field effect transistor sensors. Nano Lett. 2007, 11, 3405-3409.

24. Matsumoto, A.; Sato, N.; Sakata, T.; Yoshida, R.; Kataoka, K.; Miyahara, Y. Chemical-to-electrical-signal transduction synchronized with smart gel volume phase transition. Adv. Mater. 2009, 21, 4372-4378.

25. Matsumoto, A.; Endo, T.; Yoshida, R.; Miyahara, Y. Electrically visualized chemo-mechanical signal transduction by using a smart gel gate-modified field effect transistor. Chem. Commun. 2009, 37, 5609-5611.

26. Maeda, Y.; Matsumoto, A.; Miura, Y.; Miyahara, Y. Preparation of alpha-mannoside hydrogel and electrical detection of saccharide-protein interactions using the smart gel-modified gate field effect transistor. Nano. Res. Lett. 2012, 7, 108.

27. Matsumoto, A.; Ishii, T.; Nishida, J.; Matsumoto, H.; Kataoka, K.; Miyahara, Y. A synthetic approach toward self-regulated insulin delivery system. Angew. Chem. 2012, 51, 2124-2128.

28. Ozoemena, K.I.; Nyokong, T.; Nkos, I.D.; Chambrier, I.; Cook, M.J. Insights into the surface and redox properties od single carbon nanotube-cobalt(II) tetra-aminophthalocyanine self-assembled on gold electrode. Electrochim. Acta 2007, 52, 4132-4143.

29. Matsumoto, A.; Ikeda, S.; Harada, A.; Kataoka, K. Glucose-Responsive polymer bearing a novel phenylborate derivative as a glucose-sensing moiety operating at physiological $\mathrm{pH}$ conditions. Biomacromolecules 2003, 4, 1410-1416.

30. Otsuka, H.; Uchimura, E.; Koshino, H.; Okano, T.; Kataoka, K. Anomalous binding profile of phenylboronic acid with $\mathrm{N}$-acetylneuraminic acid (Neu5Ac) in aqueous solution with varying $\mathrm{pH}$. J. Am. Chem. Soc. 2003, 125, 3493-3502.

31. Matsumoto, A.; Matsumoto, H.; Maeda, Y.; Miyahara, Y. Simple and robust strategy for potentiometric detection of glucose using fluorinated phenylboronic acid self-assembled monolayer. BBA Gen. Subj. 2013, 1830, 4359-4364. 
32. Miyata, T.; Uragami, T.; Nakamae, K. Biomolecule-sensitive hydrogels. Adv. Drug. Deliv. Rev. 2002, 54, 79-98.

33. Qiu, Y.; Park, K. Environment-sensitive hydrogels for drug delivery. Adv. Drug. Deliv. Rev. 2001, $53,321-339$.

(C) 2014 by the authors; licensee MDPI, Basel, Switzerland. This article is an open access article distributed under the terms and conditions of the Creative Commons Attribution license (http://creativecommons.org/licenses/by/3.0/). 